\title{
Discriminability and preference in concept identification
}

\section{BRUCE D. DOWNING, Kent State University, Kent, Ohio 44240}

The hypothesis tested in this study was that the relative discriminability of dimensions would determine the rate of concept identification (CI) by determining the order of preference for testing the relevance of dimensions. A reaction time measure of discriminability and the Levine procedure for identifying hypotheses were employed. The results indicated that discriminability had no simple relationship to preference and that neither relative discriminability nor relative preference was predictive of $C I$ rates. An explanation of the results in terms of multiple hypotheses testing is offered.

The effect of selective attention variables on rate of concept identification (CI) has recently been a subject of increasing interest. The concept of selective attention implies that organisms may not attend to or respond to all aspects of the stimulus environment but only to certain selected aspects. Observations that the rate of $\mathrm{CI}$ is often dependent upon the dimension that is relevant to the solution suggests that the probability of a cue being attended to will determine, in part, the ease of $\mathrm{CI}$ when that cue is relevant. Various investigators have postulated attention factors such as discriminability (Archer, Bourne, \& Brown, 1955), obviousness (Sechrest \& Kaas, 1965; and Archer, 1962), and the ratio of the weight of relevant cues to the weight of all cues (Bourne \& Restle, 1959; Restle, 1962; and Bower \& Trabasso, 1964).

Inherent in these studies is the suggestion that the obviousness or discriminability of the dimensions of the stimuli will determine the preference of Ss for utilizing the dimensions in CI. Imai \& Garner (1965) have attempted to determine the relationship between discriminability and preference in a card-sorting task. Discriminability was defined as the physical distance between two levels of a dimension and preference was defined as the proportion of times Ss used a dimension as the basis of classification when they could employ any dimension (free classification). Their results indicated a relationship between discriminability and preference such that the greater the discriminability, the more probable it was that the dimension would be used for classification. When Ss were told what dimension to use for classification (constrained classification), only the discriminability affected the speed of classification; preference had no effect on speed.

The Imai and Garner results may not be directly applicable to a CI problem, because CI does not involve completely free nor completely constrained classification. However, if measures of discriminability and of original preference for dimensions during $\mathrm{Cl}$ are taken, observation of their effects on speed of CI would be appropriate.

The following study utilized a reaction time (RT) measure of relative discriminability and a dimension preference measure to test the hypothesis that the relative discriminability of dimensions will determine the preference for dimensions, thus determining the order of hypothesis testing and the rate of $\mathrm{Cl}$. The most discriminable dimension should be most preferred in terms of testing hypotheses about the relevance of the dimension. Therefore, $\mathrm{Cl}$ should be more rapid when the most discriminable dimension is relevant.

\section{METHOD}

Reaction Time Task

The Ss were shown a series of simul taneously presented stimuli and were required to indicate if the stimuli were the same or different. The stimuli were triangles that varied on three dichotomous dimensions: color, the vertical position of a dot in the triangle, and the number of borders inscribed in the triangles. Each $S$ saw 100 pairs of triangles. Twenty-five were the same on all dimensions; the remaining 75 were different on one of the three dimensions -25 on color, 25 on dot position, and 25 on number of borders. Prior to the task Ss were shown two triangles that differed on all three dimensions and were asked to describe the differences they observed to ensure that all dimensional differences were discriminable. Ss were then instructed to judge all pairs of stimuli either the same or different as quickly and as accurately as possible by pressing one of two buttons labeled "same" and "different." The assumption underlying this task was that the order of reacting to differences is determined by the order of discriminability, so that a difference on the most discriminable dimension should produce the fastest RT.

The stimuli were rear projected onto frosted glass with a stimulus duration of $1.5 \mathrm{sec}$ followed by a blank period of $1.5 \mathrm{sec}$. Reaction times were recorded to the nearest millisecond. The Ss used their preferred hand and the assignment of "same" and "different" buttons was counterbalanced. Thirty Ss from an introductory psychology class were employed in this part of the experiment.

Concept Identification Task

The concept identification (CI) task was a standard two-choice discrimination problem in which two stimuli were simultaneously presented and the $S$ chose the right or the left stimulus depending upon what he thought was the "correct" stimulus. The "correct" stimulus always contained the relevant level of the relevant dimension. The stimuli were again triangles exhibiting the same dimensions and levels as in the same-different (S-D) judgment task. However, in the $\mathrm{Cl}$ task the two triangles that were presented on each trial contained all levels of all dimensions. All eight combinations of the levels were employed in constructing the triangles, thus producing four different pairs. The right-left positions of the triangles were randomly assigned and stimuli were rear projected as in the S-D judgment task. $S$ pushed the right or left button on a panel to indicate his choice. Feedback was given by two lights on the response panel, a white light for correct and a blue light for incorrect. The stimulus on time was $3.5 \mathrm{sec}$ with a blank interval of $2 \mathrm{sec}$.

In order to trace the hypothesis-testing behavior of Ss and obtain a measure of preference for testing the dimensions, the Levine (1966) technique was utilized. This method included giving feedback on every fourth trial, indicating to the $S$ if he was correct or incorrect on that trial. There was no feedback on other trials but Ss were told to respond as if correct until feedback was given. This technique allows one to determine the hypothesis held by the $S$ by looking at a series of four responses.

A total of $36 \mathrm{Ss}$ from an introductory psychology course were assigned to three independent groups balanced equally for sex. Each of these groups solved a CI task with one of three dimensions relevant. The Ss were asked to describe the dimensions and levels of the stimuli before the experiment and were given a detailed set of instructions indicating how to respond and how to interpret the feedback. After the experiment Ss were asked to rate the dimensions on how obvious they were and indicate why they failed to test the most obvious dimension first when they indeed failed to do so.

\section{RESULTS}

Reaction Time Task

The last forty trials for each $S$ were used to compute a mean RT for difference judgments on color (C), dot (D), and border (B) as well as judgments of same (S). These means (in milliseconds) were as follows: $\mathrm{C}=.778, \mathrm{D}=.948, \mathrm{~B}=.997$, and $S=1.045$. According to a Newman-Keuls analysis, the difference between each pair of means was significant at either the .05 or .01 level. The interpretation is that the dimensions do differ in discriminability, with color the most discriminable, then dot position and number of borders least discriminable.

\section{Concept Identification Task}

The rate of $\mathrm{CI}$ was measured by the mean number of trials to a learning criterion of 12 correct responses. These means for D, C, and B relevant conditions were $19.33,22.67$, and 30.67 , respectively. The overall $\mathrm{F}$ for these differences was significant at the .01 level $[F(2,30)=5.531]$. A Newman-Keuls analysis on numbers of trials to solution indicated that the difference between $D$ and $B$ was significant at the .05 level and the 
difference between $C$ and $B$ at the .01 level, but the difference between $C$ and $D$ was not significant. These results indicate that $C$ and $D$ were learned faster than $B$, but $C$ and $D$ were not different in learning rate.

A measure of Ss' preference for testing the relevance of each dimension was obtained by computing the proportion of Ss who tested a dimension first. These data indicate that one-half of the Ss chose $D$ as the first dimension to test, whereas one-fourth tested $\mathrm{C}$ first and one-fourth tested $\mathrm{B}$ first. Therefore, D was initially preferred two-to-one over B and C.

The results appear to indicate that in this study discriminability as measured by RT was not the same as the order of preference. In addition, neither the order of discriminability nor the order of preference is sufficient to explain the order of $\mathrm{Cl}$ rates as a function of the relevant dimension. On the basis of the discriminability data, one would expect $C$ to have the fastest $\mathrm{Cl}$ rate, then $\mathrm{D}$ and $\mathrm{B}$ in that order. However, there was no difference in $\mathrm{Cl}$ rates for $\mathrm{C}$ and $\mathrm{D}$. The preference for $\mathrm{D}$ over $\mathrm{C}$ and $\mathrm{B}$ did not produce a CI rate for D that was faster than that of $C$, but it was faster than the rate for $B$.

One might explain these results by a combination of discriminability and preference effects. The $B$ dimension was low in preference and discriminability so that it produced the slowest $\mathrm{CI}$ rate. However, $\mathrm{C}$ was highly discriminable and D was highly preferred so that their CI rates were higher than B but not different from each other.

An insight into the nature of the preference for $D$ may be obtained from the results of Ss' verbal reports. The Ss ranked C most obvious, then D, and B least obvious, which supports the RT data. Color was judged to be the most obvious by $84 \%$ of the Ss, yet only $39 \%$ of the Ss tested color first. Among the Ss who did not test color first, $77 \%$ said that color was too obvious and, therefore, did not think it would be the relevant cue.

\section{DISCUSSION}

The results of this study indicate that one cannot accept the simple notion that the relative discriminability of dimensions will determine the relative preference for forming hypotheses on the dimensions in a CI task. Furthermore, neither measured discriminability nor preference was individually predictive of CI rates. However, one cannot reject completely the hypothesis that discriminability and preference are related, since the verbal reports of Ss indicated that color was not preferred because of its high discriminability. The low preference for color leads to the question of why the color-relevant $\mathrm{Cl}$ rate was as fast as the rate for dot, if dot was usually tested first. It is possible that Ss may have been gaining partial information about the relevance of color even when testing on dot because of the high discriminability or obviousness of color. Border was not preferred and information about its relevance may have been more difficult to abstract because of its low discriminability. At any rate, neither discriminability nor subject preference alone is sufficient to describe the process which leads to differential learning rates in concept identification.

\section{REFERENCES}

ARCHER, E J Concept identification as a function of obviousness of relevant and irrelevant information. Journal of Experimental Psychology, 1962, 63, 616-620.

ARCHER, E. J., BOURNE, L. R., \& BROWN, F. G. Concept identification as a function of irrelevant information and instructions. Journal of Experimental Psychology, 1955, 49, 153-164.

BOURNE, L. E., \& RESTLE, F. Mathematical theory of concept identification. Psychological Review, 1959, 66, 278-296.

BOWER, G. W., \& TRABASSO, T. R. Concept identification. In R. C. Atkinson (Ed.), Studies in mathematical psychology. Stanford, Calif.: Stanford University Press, 1964.

IMAI, S., GARNER, W. R. Discriminability and preference for attributes in free and constrained classification. Journal of Experimental Psychology, 1965, 69, 596-608.

LEVINE, M. Hypothesis behavior by humans during discrimination learning. Journal of Experimental Psychology, 1966, 71, 331-338.

RESTLE, F. The selection of strategies in cue learning. Psychological Review, 1962, 69, 329-343.

SECHREST, L. \& KAAS, J. S. Concept difficulty as a function of stimulus similarity. Journal of Educational Psychology, 1965, 56, 327-333. 